\title{
An Income Distribution Method for Stakeholders of Modern Apprenticeship Based on Game Theory
}

\author{
SUYAN ZHANG ${ }^{1,2}$, WEI ZHAO ${ }^{1, *}$ \\ ${ }^{1}$ College of Applied Science and Technology, Beijing Union University, Beijing, CHINA \\ ${ }^{2}$ University of Chinese Academy of Sciences, Beijing, CHINA \\ *Corresponding author: ddzhao710071@yeah.net
}

\begin{abstract}
The reasonable distribution of benefits for the stakeholders of modern apprenticeship can satisfy the interest demands of stakeholders, contribute to the construction of a modern apprenticeship system and ultimately meet the inherent requirements of vocational education for economic and social development. To solve the enterprise participation motivation and low enthusiasm, unreasonable income distribution between enterprises and colleges, this article regard enterprises and schools as the main stakeholders involved in the modern apprenticeship, using the game theory, under a non-cooperative game and cooperative game situation, analyze the cost, net earning, enterprise effort and school subsidy coefficient of modern apprenticeship, research the income distribution problem in education of modern apprenticeship between enterprises and colleges.
\end{abstract}

Keywords - modern apprenticeship, income distribution, game analysis.

Received: August 24, 2020. Revised: December 3, 2020. Accepted: December 19, 2020. Published: December 29, 2020

\section{Introduction}

$\mathrm{I}^{\mathrm{N}}$ $\mathrm{N}$ the Notice of the State Council on Printing and Distributing the "13th Five-Year" for the Development of National Educational Undertakings" ([2017] No. 4), it proposed to "actively promote the modern apprenticeship of joint enrollment and training by schools and enterprises". Moreover, in Several Opinions of General Office of the State Council on Deepening the Integration of Industry and Education" ([2017] No. 95), it pointed out that it is necessary to "deepen the reform of the school-running system in the full-time vocational schools and comprehensively implement the modern apprenticeship and new enterprise apprenticeship in technical and practical majors". However, restricted by the large investment, high cost, and unsatisfactory demand for income distribution, enterprises are short of motivation and enthusiasm to take part in[1], which makes it difficult to deepen and continue the modern apprenticeship jointly cultivated by schools and enterprises. Therefore, as the stakeholders of the modern apprenticeship, enterprises should dig out their income appeals to effectively accelerate the in-depth development of the modern apprenticeship jointly cultivated by schools and enterprises.

Modern apprenticeship is a strategic choice for vocational education to actively serve the current requirements for economic and social development, promote the interactive development of the vocational education system and the labor employment system, open up and broaden channels of training and cultivating skilled talents, and advance the construction of the modern vocational education system. Starting from the perspective of economic game, this paper analyzes stakeholders' income appeals of the modern apprenticeship, explores the source of incentives and fusion power of enterprises and schools, conducts analysis and comparation on game theory of the cultivation cost of the modern apprenticeship, net income, effort level of enterprises, and subsidy coefficients of schools under the non-cooperative game and cooperative game to provide an inherent basis for preferably coordinating the interest relationship between enterprises and institutions of higher learning and constructing a benign and orderly running system for modern apprenticeship[2].

\section{Game Elements and Assumptions of Stakeholders in the Modern Apprenticeship}

\subsection{Game Elements}

Game theory is a discipline that studies the decision-making when behaviors of the decision-making subjects directly interact and equilibrium problems of this kind of decision-making [3]. As the stakeholders of the modern apprenticeship, enterprises and institutions of higher learning will make decisions to get the maximum return in the cultivation of the modern apprenticeship in the game process based on their own information and understanding of their own abilities. Specifically, the game elements of stakeholders in the modern apprenticeship include: 
1) Participants: Participants refer to the subjects of decision-making in the game. In order to achieve their own goals, they participate in the game competition and maximize their own payment (utility) level by choosing actions (or strategies) [3]. Participants of the stakeholders' game are mainly enterprises and institutions of higher learning in the modern apprenticeship.

2) Action: The action signifies the decision of a participant at a certain point in the game. An important issue related to the action is the sequence of action [3]. For example, in the cultivation of the modern apprenticeship, if a school is the initiator and an enterprise is the responder, under the premise that there is no binding agreement regarding revenue, the school and the enterprise will be the participants in the game and conduct a sequential non-cooperative game.

3) Strategy: The strategy means that the participants will choose practical rules of action. There are two strategies for participants in the modern apprenticeship, namely, participation and non-participation. However, only when schools and enterprises participate in the modern apprenticeship can strategies be implemented, thereby reaching the research and practical significance.

4) Payment: Payment signifies the result of game competition, which is a set of strategic functions determined by the participants. Participants of modern apprenticeship will obtain a certain net benefit after choosing a participation strategy under the background of non-cooperative game and cooperative game respectively. Furthermore, net benefit is payment.

5) Equilibrium: Equilibrium refers to the optimal strategy or combination of actions of all participants. This paper analyzes the stakeholders' revenue equilibrium in the modern apprenticeship under the background of the non-cooperative game and cooperative game and makes comparations to choose equilibrium for maximizing the interests of all stakeholders.

\subsection{Game Assumptions}

In order to conduct a game analysis of stakeholders in the modern apprenticeship, assumptions are made in the followings:

1) Since schools and enterprises are the subjects of stakeholders in the modern apprenticeship as well as economically rational people with interest preferences. The relevant decision-making behaviors of both parties in the game process conform to the laws of economic rationality.

2) The value created by a student to a school is S, and the value created by the student to an enterprise is $\mathrm{E}$.

3) The training cost of the student is $\frac{1}{2} C \lambda^{2}$, of which $C$ is a constant to estimate the cost of cultivating the student, and $\lambda(0 \leq \lambda \leq 1)$ is a coefficient of effort level by the enterprise in the cultivation of modern apprenticeship. On the premise of not affecting the functional relationship between variables, the coefficient $\frac{1}{2}$ is added to the expression of student training cost in order to simplify the calculation.

4) When the enterprise completes the training work for the modern apprenticeship, the school should give the enterprise a certain training subsidy. It is assumed that the school pays the enterprise training subsidy coefficient as $t$.

T_NP,E_NP and S_NP are employed to represent the total net income of university-enterprise cooperation for the modern apprenticeship, net income of the enterprise and the net income of the school. Thereby, it can be calculated:

the total net income of university-enterprise cooperation:

$T_{-} N P=(S+E) \lambda-\frac{1}{2} C \lambda^{2}$

net income of the enterprise:

$$
E_{-} N P=E \lambda-\frac{1}{2} C \lambda^{2}(1-t)
$$

the net income of the school:

$$
S_{-} N P=S \lambda-\frac{1}{2} C \lambda^{2} t
$$

\section{Equilibrium Analysis on the Income Distribution of Stakeholders in the Modern Apprenticeship}

Since there are differences in the starting point and situation of school-enterprise cooperation, different types of game methods will be used to achieve specific cooperation goals. When the game behaviors of the school and the enterprise interact with each other for modern apprenticeship training, it is a non-cooperative game to achieve the goal of maximizing individual income if the two parties fail to reach a binding agreement on the income distribution, and it is a cooperative game to reach the goal of maximizing the total net income of the group if the two parties reach a binding agreement on the income distribution.

\section{A. 3.1. Equilibrium Analysis on the Income Distribution under the Non-Cooperative Game}

Enterprises are short of motivation and enthusiasm to take part in the modern apprenticeship jointly cultivated by schools and enterprises, which results in the difficulty of continuous improvement. Therefore, in order to primarily meet the demands of corporates' income distribution and drive them to actively participate in the cultivation of modern apprenticeship, the equilibrium analysis on the income distribution should be 
conducted to determine the school subsidies, the degree of enterprise effort, the net income of both parties and the total net income based on the non-cooperative game.

When talking about the sequence of game action, the school is usually regarded as the initiator, and the enterprise is the responder. Namely, the school promises the enterprise to provide the apprenticeship training cost and subsidy $t$, and the enterprise determines the effort level $\lambda$ after receiving the subsidy $t$, which belongs to the sequential equilibrium analysis of the non-cooperative game. Furthermore, its solution is called Stackelberg equilibrium.For this study,Stackelberg equilibrium is adopted to obtain the subsidy coefficient of the school $t$, the effort level of the enterprise $\lambda$ and the relationship between $\lambda$ and t. After Stackelberg equilibrium solution is obtained, the total net income and the net incomes of the schools and enterprise in the cultivation of the modern apprenticeship can be further acquired. Moreover, the backward induction will be used to solve Stackelberg equilibrium solution $(t, \lambda)$.

Firstly, the reaction function of $\lambda$ is determined. Since it is necessary to chiefly satisfy the income demand and ensure the maximum net income of the enterprise. As for the net income of the enterprise, the first derivative of $\lambda$ in formula (2) is calculated, and it is set to be 0 to get:

$$
\lambda=\frac{E}{C(1-t)}
$$

Secondly, the relationship between $\lambda$ and $\mathrm{t}$ is determined. Since $\frac{\partial \lambda}{\partial t}=\frac{E}{C(1-t)^{2}}>0$, the effort level of the enterprise in the modern apprenticeship is positively correlated with the school subsidy $t$. The larger $t$ is, the greater effort level of the enterprise will be. Consequently, it can be concluded that the school can pay higher subsidy to the enterprise in the income distribution of school-enterprise modern apprenticeship in order to encourage the enterprise to boost its efforts.

Thirdly, the coefficient of school subsidy $t$ and the effort level of enterprise $\lambda$ are determined, namely Stackelberg equilibrium solution. The reaction function $\lambda$, that is, formula (4) is substituted into the school net income formula (3), the first-order partial derivative of $t$ is solved, and the first-order partial derivative of $t$ is set to be 0 to ensure that the school net income is maximized. The coefficient of training subsidy in school's modern apprenticeship is gained:

$$
t^{*}=\frac{2 S-E}{2 S+E} \quad S>\frac{E}{2}
$$

Fourthly, formula (5) is substituted into formula (4), we can obtain in the effort level of the enterprise in the cultivation of modern apprenticeship $\lambda^{*}=\frac{2 S+E}{2 C}$. Therefore, when the school and the enterprise play a non-cooperative game, the Stackelberg equilibrium solution is:

$$
\left(t^{*}, \lambda^{*}\right)=\left(\frac{2 S-E}{2 S+E}, \frac{2 S+E}{2 C}\right)
$$

Finally, the incomes of all parties are solved. In the case of Stankelberg equilibrium, the total net income

$T_{-} N P^{*}=\frac{(S+E)(2 S+E)}{2 C}-\frac{(2 S+E)^{2}}{8 C}$; the net income of the enterprise $E_{-} N P^{*}=\frac{E(2 S+E)}{2 C}-\frac{(2 S+E)^{2}(1-t)}{8 C}$; and the net income of the school $S_{-} N P^{*}=\frac{S(2 S+E)}{2 C}-\frac{(2 S+E)^{2} t}{8 C}$.

\subsection{Equilibrium Analysis on the Income Distribution under the Cooperative Game}

In order to enable the modern apprenticeship jointly cultivated by the school and enterprise to continuously advance, it should take not only the individual income but also the incomes of all stakeholders into consideration. Therefore, in order to increase the income of the school and the enterprise, or at least enhance the income of one party without prejudice to the income of the other party, the equilibrium analysis on the income distribution can be conducted based on the cooperative game. Under the premise of maximizing the total net income of school-enterprise cooperation, the income constraints can be formulated, and the school subsidy $t$ and the enterprise's effort level $\lambda$ can be determined. In order to maximize the total net income under the cooperative game, the total net income of school-enterprise cooperation, $\lambda$ in the formula (1), should be adopted to calculate the first-order partial derivative, and the value should be appointed as 0 . Therefore, the enterprise's

effort level $\lambda^{*}=\frac{S+E}{C}$ can be calculated. In the equilibrium of the cooperative game, the total net income is $\overline{T \_N P^{*}}=\frac{(S+E)^{2}}{C}-\frac{(S+E)^{2}}{2 C}=\frac{(S+E)^{2}}{2 C}$; the net income of the enterprise is $\overline{E_{-} N P^{*}}=\frac{(S+E) E}{C}-\frac{(S+E)^{2}(1-t)}{2 C}$; the net income of the school is $\overline{S_{-} N P^{*}}=\frac{S(S+E)}{C}-\frac{(S+E)^{2} t}{2 C}$. The equilibrium solution set of the income distribution of the cooperative game should satisfy:

$N\left(\overline{t^{*}}, \overline{\lambda^{*}}\right)=\left\{\left(\overline{t^{*}, \overline{\lambda^{*}}}\right) \mid \overline{S_{-} N P^{*}}\left(\overline{t^{*}}, \overline{\lambda^{*}}\right) \geq S_{-} N P^{*}, \overline{E_{-} N P^{*}}\left(\overline{t^{*}}, \overline{\lambda^{*}}\right) \geq E_{-} N P^{*}\right\}$

The solution set indicates that the net income of the school and the enterprise under the cooperative game should be greater than or equal to the net income of the school and the enterprise under the non-cooperative game, namely:

$$
\begin{aligned}
& \Delta S_{-} N P=\overline{S_{-} N P^{*}}-S_{-} N P^{*}=\frac{S(S+E)}{C}-\frac{(S+E)^{2} t}{2 C}-\frac{S(2 S+E)}{2 C}+\frac{(2 S+E)^{2} t}{8 C} \geq 0 \\
& \Delta E_{-} N P=\overline{E_{-} N P^{*}}-E_{-} N P^{*}=\frac{(S+E) E}{C}-\frac{(S+E)^{2}(1-t)}{2 C}-\frac{E(2 S+E)}{2 C}+\frac{(2 S+E)^{2}(1-t)}{8 C} \geq 0
\end{aligned}
$$

The scope of school subsidy can be obtained by formulas (8) and (9), that is $\frac{4 S-E}{4 S+3 E} \leq t^{*} \leq \frac{4 S}{4 S+3 E}$. Therefore, in the case of the cooperative game, the equilibrium solution set of the game is: 
$N\left(\overline{t^{*}}, \overline{\lambda^{*}}\right)=\left\{\left(\overline{t^{*}}, \overline{\lambda^{*}}\right) \mid \frac{4 S-E}{4 S+3 E} \leq t^{*} \leq \frac{4 S}{4 S+3 E}, \overline{\lambda^{*}}=\frac{S+E}{C}\right\}$. It can be seen that $N\left(\overline{t^{*}}, \overline{\lambda^{*}}\right)$ is non-empty, manifesting that the enterprise and the school can obtain more income than that in the case of the non-cooperative game. Moreover, the residual income is recorded as $\Delta T_{-} N P=\overline{T_{-} N P^{*}}-T_{-} N P^{*}=\frac{E^{2}}{8 C}$. Consequently, it can be concluded that the income of the cooperative game is greater than that of the non-cooperative game, and schools and enterprises should select cooperative games for cultivation.

The residual income can be distributed between the school and enterprise. Based on the bargain model, a unique result of the subgame-perfect equilibrium can be obtained from the equilibrium solution set of the cooperative game, that is, the only solution that determines the school subsidy and the effort level of the enterprise. The residual income can be allocated through negotiation and bargaining between the school and the enterprise. It can be analyzed according to Rubinstein's bargain model. The unique result of the subgame-perfect equilibrium can be calculated in alternation offers of indefinite duration $r^{*}=\frac{1-\delta_{2}}{1-\delta_{1} \delta_{2}}$ [4]. Among them, $\delta_{1}$ represents the patience level of the enterprise, and $\delta_{2}$ represents the patience level of the school. The patience level hinges on the risk preference and negotiation cost of the school and the enterprise. The party with higher patience level can achieve larger residual income. When $\delta_{1}$ and $\delta_{2}$ are known, the residual income obtained by the enterprise is $\Delta E_{-} N P=\left(1-r^{*}\right) \Delta T T_{-} N P=\frac{\delta_{2}\left(1-\delta_{2}\right) E^{2}}{8\left(1-\delta_{1} \delta_{2}\right) C}$; the residual income obtained by the school is: $\Delta S_{-} N P=r^{*} \Delta T_{-} N P=\frac{\left(1-\delta_{2}\right) E^{2}}{8\left(1-\delta_{1} \delta_{2}\right) C}$, and the coefficient of school subsidy is

$\overline{t^{*}}=t_{\max }-\frac{\Delta S \_N P}{\overline{Y^{*}}}=t_{\min }+\frac{\Delta E \_N P}{\overline{Y^{*}}}=\frac{4 S-E}{4 S+3 E}+\frac{\delta_{2}\left(1-\delta_{2}\right) E^{2}}{4\left(1-\delta_{1} \delta_{2}\right)(S+E)^{2}}$. The lower limit and upper limit of school subsidy and student training cost are $t_{\min }=\frac{4 S-E}{4 S+3 E}, t_{\max }=\frac{4 S}{4 S+3 E}$ and $\overline{Y^{*}}=\frac{1}{2} C \bar{\lambda}^{*^{2}}=\frac{(S+E)^{2}}{2 C}$ respectively. Therefore, in the cooperative game between the school and the enterprise, the only equilibrium solution is

$$
\left(\overline{t^{*}}, \overline{\lambda^{*}}\right)=\left(\frac{4 S-E}{4 S+3 E}+\frac{\delta_{2}\left(1-\delta_{1}\right) E^{2}}{4\left(1-\delta_{1} \delta_{2}\right)(S+E)^{2}}, \frac{S+E}{C}\right)
$$

\section{Equilibrium Simulation of Income Distribution Among Stakeholders in the Modern Apprenticeship}

\subsection{Estimation Method of Patience Level of Income Distribution Equilibrium}

Through the above analysis, it can be found that the income of modern apprenticeship in the cooperative game are higher than that in the non-cooperative game. The distribution of residual income in the cooperative game and the determination of the unique equilibrium solution depend on the confirmation of the patience level of the school and enterprise. This paper adopts fuzzy comprehensive evaluation to ascertain the patience coefficient of the school and enterprise. The patience level is mainly affected by three factors, including industry status, negotiation cost and risk preference. The set of factors should be firstly defined: $U=\{$ industry status, negotiation cost, risk preference $\}$. According to the varied degrees of influence, the weight vector $F=\{f 1, f 2, f 3\}$ of the three factors should be defined, and the evaluation set of each factor should be illustrated as $\mathrm{V}=\{$ lowest, lower, medium, higher, highest $\}$, and its corresponding quantity value is $\mathrm{V}$ $=\{0.1,0.3,0.5,0.7,0.9\}$, which implies the corresponding relationship between each element of the evaluation set and the value of the patience coefficient[4]. Secondly, the fuzzy relation matrix from $\mathrm{U}$ to $\mathrm{V}$ should be determined. After expert evaluation, the patience coefficient should be evaluated to form a correspondence table for the different evaluation degrees of the three factors (see Table 1). Moreover, three factors affecting patience level should be estimated according to the five levels of the evaluation set. With the integration of the evaluation results by all experts, the evaluation result of each level of each factor is converted into the numerical value in [0, 1] [4]. Through matrix calculation, three fuzzy vectors of factors affecting patience level are obtained: $\mathrm{F}$ industry status, $\mathrm{F}$ negotiation cost, and $\mathrm{F}_{\text {risk preference. }}$

Table 1 Evaluation Criteria of Patience Level

\begin{tabular}{c|c|c|c|c|c}
\hline Patience Level & 0.1 & 0.3 & 0.5 & 0.7 & 0.9 \\
\hline Industry Status & & & & Higher & Highest \\
\hline Negotiation cost & Lowest & Lower & Medium & Lower & Lowest \\
\hline Risk preference & Highest & Higher & Medium & Higher & Highest \\
\hline
\end{tabular}


The above three evaluation results are combined into a matrix to obtain the fuzzy relation matrix from $\mathrm{U}$ to $\mathrm{V}$ :

$$
W=\left\lfloor\begin{array}{l}
F_{\text {industry status }} \\
F_{\text {negotiation cost }} \\
F_{\text {risk preference }}
\end{array}\right\rfloor=\left[\begin{array}{lllll}
W_{11} & W_{12} & W_{13} & W_{14} & W_{15} \\
W_{21} & W_{22} & W_{23} & W_{24} & W_{25} \\
W_{31} & W_{32} & W_{33} & W_{34} & W_{35}
\end{array}\right]
$$

fuzzy comprehensive evaluation is carried through

$$
D=F \times W=\left[\begin{array}{lll}
f_{1} & f_{2} & f_{3}
\end{array}\right] \times\left[\begin{array}{lllll}
W_{11} & W_{12} & W_{13} & W_{14} & W_{15} \\
W_{21} & W_{22} & W_{23} & W_{24} & W_{25} \\
W_{31} & W_{32} & W_{33} & W_{34} & W_{35}
\end{array}\right]=\left[\begin{array}{lllll}
d_{1} & d_{2} & d_{3} & d_{4} & d_{5}
\end{array}\right]
$$

After normalization processing:

$$
D^{\prime}=\left\lfloor\begin{array}{lllll}
d_{1}^{\prime} & d_{2}^{\prime} & d_{3}^{\prime} & d_{4}^{\prime} & d_{5}^{\prime}
\end{array}\right\rfloor
$$

Finally, the coefficient of patience level is calculated as:

$$
\delta=D^{\prime} \times V^{T}=\left[\begin{array}{lllll}
d_{1}^{\prime} & d_{2}^{\prime} & d_{3}^{\prime} & d_{4}^{\prime} & d_{5}^{\prime}
\end{array}\right] \times\left[\begin{array}{c}
0.1 \\
0.3 \\
0.5 \\
0.7 \\
0.9
\end{array}\right]=0.1 d_{1}^{\prime}+0.3 d_{2}^{\prime}+0.5 d_{3}^{\prime}+0.7 d_{4}^{\prime}+0.9 d_{5}^{\prime}
$$

\section{A. 4.2. Verification of Income Distribution equilibrium}

Firstly, parameters are defined for enterprises and schools to jointly carry out training for the modern apprenticeship: $S=50$ ; $\mathrm{E}=50 ; \mathrm{t}=4 ; \mathrm{C}=1000$.

Secondly, according to the estimation method of patience level, the patience coefficient of the enterprise is calculated. According to "Table 1 Evaluation Criteria of Patience Level ", the comprehensive fuzzy relation matrix is:

$$
W=\left[\begin{array}{ccccc}
0.5 & 0.1 & 0.2 & 0.2 & 0 \\
0.2 & 0.3 & 0.3 & 0.1 & 0.1 \\
0.3 & 0.1 & 0.1 & 0.2 & 0.3
\end{array}\right]
$$

Thirdly, the corresponding weight vector $\mathrm{F}=\left[\begin{array}{lll}0.4 & 0.3 & 0.3\end{array}\right]$ is assumed for each factor, and fuzzy comprehensive evaluation is performed to get $\mathrm{D}=\mathrm{F} \times \mathrm{W}=\left[\begin{array}{lllll}0.35 & 0.16 & 0.2 & 0.17 & 0.12\end{array}\right]$, which indicates that the five patience levels ("lowest", "lower", "medium", "higher", and "highest") accounting for 0.35, 0.16, $0.2,0.17$ and 0.12 respectively, calculating the patience coefficient of the enterprise :

$$
\delta_{1}=D^{\prime} \times V^{T}=\left[\begin{array}{lllll}
0.35 & 0.16 & 0.2 & 0.17 & 0.12
\end{array}\right] \times\left[\begin{array}{l}
0.1 \\
0.3 \\
0.5 \\
0.7 \\
0.9
\end{array}\right]=0.41
$$

Finally, the above method is applied to the calculation of the fuzzy relation matrix for school's patience level, and the school's patience coefficient is further calculated $\delta_{2}=0.35$. On this basis, the result of subgame-perfect equilibrium is obtained:

$r^{*}=\frac{1-\delta_{2}}{1-\delta_{1} \delta_{2}}=0.759$

After the above parameters are acquired, they are substituted into the solution set of the Stackelberg equilibrium and the cooperative game equilibrium respectively to obtain the non-cooperative game $\left(t^{*}, \lambda^{*}\right)=(0.33,0.075)$ and the cooperative game $\left(t^{*}, \lambda^{*}\right)=(0.44,0.1)$. It can be observed that, with the increase of the school's subsidy to the enterprise, the training effort of the enterprise in modern apprenticeship has also upgraded, which is consistent with the analysis of income distribution under the non-cooperative game.

The net incomes of the non-cooperative game and the cooperative game are calculated respectively. Under the non-cooperative game, the total net income is $T_{-} N P^{*}=4.7$, the net income of the enterprise is $E_{-} N P^{*}=1.9$, and the net income of the school is $S_{-} N P^{*}=2.8$. Under the cooperative game, the total net income is $T N P^{*}=5$, the net income of the enterprise is $E_{-} N P^{*}=2.2$, and the net income of the school is $S_{-} N P^{*}=2.8$. Obviously, the total net income and the net income of the enterprise when the school and enterprise are engaged in the cooperative game are higher than those when the school and enterprise are engaged in the non-cooperative game. Namely, the cooperative game between the two parties can create more income than the non-cooperative game, which agrees with the analysis of income distribution under the cooperative game.

\section{Conclusions on the benefit distribution of modern apprenticeship stakeholders}

Modern apprenticeship is a process in which the subjects of stakeholders play games and seek win-win situation. Since stakeholders have different income demands, they have various contradictions and conflicts in the cooperation process [5], which makes it difficult to deepen and continue the modern apprenticeship system jointly cultivated by schools and enterprises, and urgently needs a reasonable mechanism to coordinate. By virtue of employing game theory to analyze the income distribution of modern apprenticeship in school-enterprise cooperation, all parties' income demands can be satisfied, and modern apprenticeship in school-enterprise 
cooperation can be promoted in the long-term and orderly development.

In the first place, through the analysis on the income equilibrium in the non-cooperative game, it is found that the school can provide higher training subsidy to the enterprise to profoundly enhance the effort level and enthusiasm of the enterprise to participate in the cultivation of the modern apprenticeship, upgrading the total net income of school-enterprise cooperation in the cultivation of the modern apprenticeship.

In the second place, when analyzing the income distribution equilibrium of the cooperative game, it is discovered that the total net income of the modern apprenticeship training in the cooperative game is higher than that in the non-cooperative game. Therefore, under the premise of maximizing the total net income, the enterprise and the school should chiefly adopt the cooperative game and formulate a binding agreement of income distribution to ensure that the income of the school and enterprise increases, thereby boosting the total net income of training.

Last but not least, the residual income generated in the cooperative game can be analyzed and allocated under Rubinstein's bargain model. The allocation process depends on the patience level of the school and enterprise in the cultivation of the modern apprenticeship, which will be affected by industry status, negotiation cost and risk preference. The higher the patience level is, the more residual income will be.

\section{Acknowledgment}

This study were supported by the "Premium Funding Project for Academic Human Resources Development in Beijing

Union University".

\section{References}

[1] Liao Liping. Current Situation, Problems and Countermea sures of the Training model in the Modern Apprenticeship [J]. Vocational \& Technical Education Forum, 2019(6):13 4-139.

[2] Wu Pingping. Research on the Stakeholders of the Modern Apprenticeship Based on the Perspective of the Cooperati ve Game[J]. Education and Occupation, 2018, No.918(14) :7-13.

[3] Zhang Weiying. Game Theory and Information Economic s [M]. Shanghai People's Publishing House. 2004:27.

[4] Bao Xinzhong, Wang Daoping. Game Analysis of Innovat ion Cost Apportionments and Income Distribution of Indu stry-University-Research Cooperation [J]. R\&D Managem ent, 2010(05):81-87.
[5] Wang Qin, Li Huifeng. Construction of a Long-Term Mec hanism for School-Enterprise Cooperation Based on the $\mathrm{C}$ ooperative Game [J]. Chinese Vocational and Technical E ducation, 2014(36): 24-29.

\section{Creative Commons Attribution License 4.0 (Attribution 4.0 International, CC BY 4.0)}

This article is published under the terms of the Creative Commons Attribution License 4.0 https://creativecommons.org/licenses/by/4.0/deed.en US 